ÉGYPTE monde arabe

\section{Égypte/Monde arabe}

18-19| 1994

L'éducation en Égypte

\title{
Le travail des enfants en Égypte et ses rapports avec la scolarisation. Esquisse d'évolution
}

\section{Nadia Zibani}

\section{(2) OpenEdition}

\section{Journals}

Édition électronique

URL : https://journals.openedition.org/ema/95

DOI : $10.4000 /$ ema. 95

ISSN : 2090-7273

\section{Éditeur}

CEDEJ - Centre d'études et de documentation économiques juridiques et sociales

\section{Édition imprimée}

Date de publication : 30 septembre 1994

Pagination : 135-167

ISSN : 1110-5097

\section{Référence électronique}

Nadia Zibani, «Le travail des enfants en Égypte et ses rapports avec la scolarisation. Esquisse d'évolution », Égypte/Monde arabe [En ligne], 18-19 | 1994, mis en ligne le 08 juillet 2008, consulté le 07 juillet 2022. URL : http://journals.openedition.org/ema/95 ; DOI : https://doi.org/10.4000/ema.95

Ce document a été généré automatiquement le 7 juillet 2022.

Tous droits réservés 


\title{
Le travail des enfants en Égypte et ses rapports avec la scolarisation. Esquisse d'évolution
}

\author{
Nadia Zibani
}

1 S'il existe, de nos jours encore, un phénomène qui dérange les pouvoirs publics, que dénoncent les instances internationales et qui révolte l'opinion, c'est bien !a persistance de la mise au travail des enfants à un âge précoce, pratique qui a presque disparu des pays industrialisés mais qui demeure fréquente dans les pays en développement ${ }^{1}$. En effet, pour certains, l'éradication de ce «fléau » doit passer par l'élaboration puis l'application draconienne d'un dispositif législatif réglementant le travail des enfants. Pour d'autres, plus que par une législation visant à le combattre, c'est avant tout par la scolarisation obligatoire et gratuite que cette pratique peut diminuer. Or, jusqu'à présent, force est de constater que, ni la législation d'un côté, ni la scolarisation obligatoire de l'autre, n'ont réussi à enrayer le phénomène.

2 Aujourd'hui, dans les sociétés occidentales, la grande majorité des enfants ne participe plus que de manière marginale aux activités productives et, à certains âges généralement entre 6 et 15 ans - ils ont pour caractéristique commune d'être scolarisés et donc de ne pas exercer d'activité économique. La réalité est bien différente dans les pays du tiers-monde, où nombreux sont les enfants qui entrent dans une vie active précoce. Qu'il s'agisse d'activités rémunérées ou non choisies par l'enfant ou à sa place, exercées sous la tutelle des parents, d'un tiers ou de façon autonome, la participation économique des enfants est loin d'être une pratique isolée et marginale. Dans un pays comme l'Égypte, où les opportunités d'emploi offertes aux enfants sont nombreuses et d'accès relativement facile - surtout dans le secteur informel, où l'éducation représenterait un investissement à long terme impliquant des coûts importants, et où le chômage touche de plus en plus des diplômés primo-demandeurs d'emploi -, le recrutement d'un nombre croissant d'enfants peut-il être considéré comme un phénomène «normal » et prévisible ? C'est sans doute à juste titre que, dans la plupart des études académiques, l'on considère le taux d'activité des enfants comme 
une variable dépendant du niveau de revenu des familles (et de la taille de ces dernières). Il nous paraît néanmoins erroné de faire du taux de scolarisation une variable dépendant du travail des enfants. Un examen attentif des statistiques de l'emploi au cours des dix dernières années montre que ce phénomène est une réalité stable en Égypte: le taux d'activité des 6-14 ans varie, au cours de cette période, autour de ce que l'on pourrait considérer comme un "seuil incompressible» de $12 \%$. Ce qui n'a pas empêché le taux de scolarisation des enfants de cet âge, comme le montrent les statistiques du Ministère de l'Education, de connaître une hausse importante durant cette même période.

$3 \mathrm{Si}$, pendant longtemps, on a implicitement traité la relation travail-école en termes de «vases communicants» - la scolarisation des enfants diminuant avec leur mise au travail -, c'est que, d'une manière générale, les taux d'activité des moins de 15 ans sont inversement proportionnels aux taux d'inscription scolaire. Or, malgré les efforts déployés en faveur de l'éducation, tout se passe comme si coexistaient simultanément la stabilité du taux d'activité des enfants et l'augmentation rapide du taux des inscriptions scolaires de la population en âge de fréquenter l'école obligatoire.

Dans ce qui suit, je me propose d'examiner l'idée de concurrence ou de compatibilité entre deux activités a priori inconciliables, le travail et l'école, chez les enfants âgés de 6 à 14 ans. Désormais, l'école va de soi pour beaucoup et l'existence d'enfantsproducteurs est l'objet d'une large désapprobation, même si leur appréhension par l'appareil statistique constitue en quelque sorte la reconnaissance de leur existence. Comme on le verra plus loin, l'absence de liaison inverse entre activité productive et activité scolaire témoigne du manque d'harmonie et/ou de pertinence dans les définitions et les concepts habituellement utilisés à ce sujet et incite à se pencher plus particulièrement sur les «oubliés » de la statistique, qui relèvent de catégories telles que : les enfants producteurs et écoliers ou les enfants inactifs et non scolarisés, qui représentent sans doute en Égypte des effectifs relativement importants.

Dans un premier temps, je présenterai une esquisse de cadre conceptuel et méthodologique d'analyse, après discussion sur les diverses catégories habituellement employées et examen des différentes combinaisons possibles entre ces dernières. Puis, j'ébaucherai une rétrospective, jusqu'en 1917, de l'évolution des taux d'activité des enfants de 6-14 ans, en distinguant le sexe et deux sous-groupe d'âges, après avoir examiné les différents problèmes techniques posés par cette opération. Enfin, sur la base des résultats disponibles de l'enquête sur l'emploi de 1988 (la plus complète à ce jour), j'examinerai quelques-unes des relations (de complémentarité ou de concurrence) existant entre travail et scolarisation des enfants.

Cadre conceptuel et méthodologiqueA propos du "sentiment de l'enfance »

6 Dans les sociétés rurales occidentales dites «traditionnelles", on a longtemps considéré comme "normal» que les enfants soient associés aux activités agricoles ou domestiques et que l'apprentissage précoce d'un métier s'effectue aux côtés des adultes, ce qui contribuait à la nécessaire socialisation enfantine. Un détour par l'histoire nous permet de constater que le travail des enfants est un "problème " relativement récent. Si nous nous défaisons quelques instants de nos habitudes de pensée contemporaines, nous observons qu'avec la naissance, au XIX ${ }^{e}$ siècle, de l'idée du caractère singulier de l'enfant ${ }^{2}$, va naître la perception du travail des enfants comme phénomène spécifique et posant problème. Dès lors, l'enfant fait l'objet d'une attention particulière, que ce soit au niveau familial, éducatif ou social. Le statut d'« enfant » 
commence à changer et, progressivement, ce dernier va être retiré de la sphère productive pour être pris en charge et formé par la famille et par des institutions comme l'école. Dès la fin du xıx siècle, en Occident, se mettent peu à peu en place des lois qui réglementent l'âge minimum d'admission au travail et l'âge limite de scolarité obligatoire, imposant petit à petit l'idée d'un antagonisme radical entre la notion d'enfance et la notion de travail. C'est donc la conception selon laquelle l'enfant doit être improductif et n'avoir d'autre "tâche » que de se former, qui prévaut aujourd'hui. De fait, c'est bel et bien cette conception qui est reprise dans les textes de lois et les conventions internationales. Si, au niveau des principes, elle tend à s'imposer comme norme juridique universelle et si, dans les mentalités, elle semble gagner du terrain, dans la pratique en revanche, il en va tout autrement: dans de nombreux pays, la proportion d'enfants qui travaillent reste importante, quand elle ne croît pas purement et simplement.

7 De l'enfance à l'âge adulte, dans des contextes socio-culturels différents (à l'intérieur d'une même société ou entre sociétés diverses), on assiste à des variations considérables dans l'enchaînement des étapes du cycle de vie (scolarisation, mise au travail, mariage, etc.). De ce fait, on peut affirmer que l'âge biologique n'a, en lui-même, aucune signification sociale universelle. Seul un ethnocentrisme aveugle nous permettrait d'affirmer que l'enfance ne se définit que par l'âge et que cet âge est le même dans toutes les sociétés. Il faut donc récuser la vision trop mécaniste qui consiste à associer systématiquement les mêmes attributs à tous les individus du même âge.

8 Souvent, dans les pays où les ruraux représentent une proportion importante de la population, la période impartie à la «jeunesse " est très courte et se confond avec l'enfance, car la mise au travail intervient très tôt. A l'opposé, dans les villes, où l'application des réglementations est plus stricte - le maintien dans le système scolaire y est supposé, en principe, mieux respecté - la période de la « jeunesse » a tendance à être plus longue. Si c'est l'aspect juridique qui est retenu comme définition de l'enfant, à savoir «toute personne n'ayant pas atteint l'âge de 15 ans », la fixation de cette limite supérieure comme critère de définition de ce sous-groupe paraît répondre à une nécessité de la comparaison statistique et a incontestablement quelque chose d'artificiel.

9 Si, de manière générale, dans les pays en développement, la proportion des moins de 15 ans dans la population est importante, ces derniers ne doivent pas nécessairement être tous assimilés à des inactifs - en l'occurrence, à des écoliers potentiels. L'apprentissage "sur le tas", principalement dans le secteur informel, constitue un facteur important du processus de formation et peut parfois s'effectuer en parallèle et en complément de l'enseignement scolaire; c'est pourquoi il faut se garder de considérer les effectifs des moins de 15 ans comme une simple mesure du nombre d'inactifs à la charge de ces économies. Par conséquent, si désormais, dans les sociétés occidentales, travail et école parmi les moins de 15 ans sont de fait des activités exclusives, il semble que dans le cas égyptien, loin d'être un phénomène marginal, la combinaison travail-école trouve un terrain favorable, même si, dans ce cas précis, l'outil statistique a bien du mal à saisir cette réalité concrète. Ainsi, le refus de principe d'accepter l'idée que des enfants puissent travailler conduit souvent à négliger deux types d'hypothèses: des enfants actifs peuvent être scolarisés et des inactifs ne pas l'être.

Méthodologie et définition des statuts des 6-14 ans 
10 Pour les appareils statistiques de la plupart des pays, un individu ne peut être considéré comme actif qu'à partir de l'âge de 15 ans. Quel que soit le seuil fixé pour définir l'âge actif, les individus l'ayant atteint sont classés soit comme actifs, soit comme inactifs, l'inactivité étant strictement définie comme la situation des élèves, des étudiants, des femmes au foyer, des retraités et des invalides. Toute personne considérée comme active est soit occupée, soit à la recherche d'un emploi. Dans le double cadre, d'une part, d'un seuil d'âge actif légal fixé à 15 ans et repris comme tel par l'appareil statistique et, d'autre part, d'une scolarité obligatoire jusqu'à cet âge, les enfants de moins de 15 ans qui ne sont ni élèves ni invalides devraient en toute rigueur être classés dans une catégorie intitulée "valide non scolarisé", la plupart du temps inexistante dans les nomenclatures statistiques. S'agissant de l'Égypte, le chercheur se trouve devant le cas favorable d'un appareil statistique qui découple l'âge minimum légal de mise au travail et la nécessité de prendre en compte la pratique réelle : le seuil d'âge actif «statistique » a donc été fixé à 6 ans.

11 Un premier obstacle à l'appréhension du travail des enfants est donc théoriquement écarté. Cependant, s'agissant de certaines catégories d'enfants, le problème de la définition du travail professionnel reste posé ; il s'apparente à celui de la définition du travail féminin dans le cadre d'unités familiales de production, où les temps et espaces d'activité domestique se confondent avec les temps et espaces d'activités productives ou s'imbriquent mutuellement. En fait - et pour simplifier - si la notion d'aide familiale (ou de travailleur familial non rémunéré, féminin et/ou enfantin) était rigoureusement définie et utilisée dans les recensements et enquêtes, le problème de l'appréhension et de la mesure du travail des enfants serait en grande partie résolu. Dans la mesure où ce n'est pas totalement le cas en Égypte, on peut seulement affirmer que ce que mesurent les recensements et les enquêtes sur l'emploi constitue ce que l'on pourrait assimiler à un "noyau dur » du travail précoce, correspondant aux critères habituels de saisie des actifs et de l'activité. Par ailleurs, le fait que la majorité des enfants actifs travaillent dans le secteur dit informel contribue probablement à renforcer encore la sous-déclaration de leur activité. Enfin, échappent à l'enregistrement statistique des activités prohibées (qui leur sont légalement interdites, par exemple la prostitution), ou non prises en compte par la nomenclature (dont le statut d'activité productive n'est pas envisagé par l'appareil statistique). Force est donc de constater que la mesure de l'activité des jeunes travailleurs est loin d'être parfaitement rigoureuse.

12 Cependant, les enfants de moins de 15 ans ne sont pas, comme tendent à les présenter les statistiques de l'éducation ou de l'emploi, constitués de groupes à statut unique, en l'occurrence d'écoliers ou d'actifs; l'observation de la réalité nous offre une variété de cas de figure qui ne rentrent pas dans le cadre dichotomique habituellement retenu opposant écolier à actif. Il existe en effet un statut mixte qui associe les deux termes de cette dichotomie (certains enfants sont à la fois scolarisés et au travail) et il en existe un autre qui les exclut tous les deux (certains enfants ne sont ni scolarisés ni actifs).

Pour étudier comment les 6-14 ans se répartissent entre les quatre statuts qui viennent d'être définis, il pourrait sembler suffisant de comparer les données démographiques globales concernant les classes d'âge retenues et extraites des recensements de population, les données concernant la scolarisation des enfants et d'autres concernant la population active. En réalité, l'analyse comparative est loin d'être aussi simple, car les trois types de statistiques requises (démographie, éducation et emploi) sont le 
produit de définitions et de traitements différents, que l'on va envisager dans le cadre de l'examen successif des quatre cas de figure définis ci-dessus.

Premier cas de figure

14 Selon la loi 139 de 1981 portant sur la scolarité obligatoire (ta'lîm al-asâsi), celle-ci s'étale sur huit années (cinq en primaire, trois en préparatoire) et l'âge normal d'entrée à l'école est fixé à 6 ans. Même si l'inscription scolaire n'est pas toujours synonyme de fréquentation effective, l'enfant inscrit dans une école est enregistré comme écolier sur les registres du Ministère de l'Education (la collecte des données auprès des établissements ayant lieu le 15 novembre de l'année scolaire) et compté dans la statistique scolaire de ce dernier, très partiellement publiée. Dans ce cas, l'enfant fait partie de la population d'âge scolaire, c'est-à-dire de «tous les individus appartenant à des groupes d'âges donnés que la loi oblige ou qui sont admis à fréquenter l'école à un niveau donné $»^{3}$. En Égypte, le groupe d'âge fréquentant normalement le primaire est celui des 6-11 ans, et celui correspondant au préparatoire est le groupe des 12-14 ans. A côté de cette première source statistique, il en existe une autre, issue des recensements de population. Dans la nomenclature de ces derniers concernant le type d'activité, le statut d'« écolier à plein temps " constitue l'un des items du sous-ensemble intitulé "population inactive ", qui inclut également des catégories comme les étudiants, les femmes au foyer, les retraités et les invalides. Le mode de recueil des données ne prévoit malheureusement pas l'enregistrement de plus d'un item, par exemple le statut simultané d'écolier et d'actif.

On comparera ici, à titre d'exemple, les effectifs d'enfants scolarisés dans le primaire et le préparatoire donnés par le recensement de novembre 1976 et ceux donnés pour l'année scolaire 1976-77 par le Ministère de l'Education. Selon la première source, le nombre d'élèves scolarisés était de 5.483 .000 et, selon la seconde, le nombre d'élèves inscrits était de 5.587.000, soit une différence de 104.000. On peut supposer que cette différence correspond en partie à des enfants inscrits à l'école mais qui ne sont pas déclarés par le chef de famille comme élèves au moment du recensement, donc à des inscrits ne fréquentant pas l'école; dans ce cas, ne s'agit-il pas d'élèves "potentiellement actifs»?

Second cas de figure

A l'opposé, si l'individu n'est pas scolarisé au moment du recensement ou de l'enquête emploi (qu'il l'ait été ou non auparavant) et exerce uniquement une activité productive, il doit, en principe, être compté dans la catégorie "population active» constituée, en Égypte, par la population âgée de 6 ans et plus, occupée ou au chômage, c'est-à-dire exerçant une activité économique ou étant à la recherche d'un emploi.

Il faut rappeler que, même s'agissant des enfants, le statut dans la profession peut être de plusieurs types: salarié, apprenti, aide familial et même indépendant, ce que l'on oublie trop souvent (vendeurs de journaux, cireurs de chaussures, etc.). Les recensements et enquêtes emploi donnent, au sujet des enfants, des informations de ce type. Malheureusement, ils ne fournissent aucune donnée ni sur la régularité (travail saisonnier ou continu); ni sur l'intensité du travail (à mi-temps, à plein temps, etc.). Par contre, les données de RP (recensements de population) permettent de distinguer les activités rémunérées de celles qui ne le sont pas (notons toutefois l'ambiguïté, sous ce rapport, du statut d'apprenti). Enfin, si l'on connaît par les RP, pour les 6-14 ans, la branche d'activité, la profession n'est pas précisée (elle ne l'est que pour les plus de 15 ans). 

définition suivante (sans doute en partie contestable, comme toute définition concernant ce domaine) : "Est enfant travailleur toute personne, garçon ou fille, de moins de 15 ans, engagée dans une activité économique au détriment des activités éducatives et ludiques considérées comme normales pour les enfants de cette tranche d'âge ».

Troisième cas de figure

19 constituent des catégories exclusives l'une de l'autre. Cet implicite semble commun à l'opinion publique et à la plupart des appareils statistiques qui, habituellement, ne comptabilisent les moins de 15 ans qu'en tant qu'écolier ou (exclusif) travailleur. Si la double activité (productive et scolaire) constitue un problème "idéologique », sa prise en compte par la statistique pose en outre un problème technique... qui ne semble plus guère en être un : à preuve les enquêtes emploi sur échantillon, pratiquées désormais dans beaucoup de pays en développement, qui posent très explicitement des questions sur les caractéristiques non seulement de l'activité principale mais aussi sur celles d'une éventuelle seconde - voire troisième - activité. S'agissant du groupe des 6-14 ans, on peut tout à fait concevoir, dans le même état d'esprit, qu'à la suite des questions portant sur l'activité principale (école ou travail), soient envisagées des questions complémentaires portant sur l'exercice parallèle éventuel d'une deuxième activité. Une enquête sur l'emploi, celle d'octobre 1988 (par ailleurs considérée comme la meilleure) a d'ailleurs envisagé la concomitance de ces deux formes d'activités. deux activités. Mais s'il veut cerner le phénomène de façon globale, c'est-à-dire au niveau statistique, il se heurte, en l'absence de sources statistiques adéquates, aux plus grandes difficultés. En effet, comme on l'a vu, la statistique scolaire n'enregistre que des inscrits (non nécessairement scolarisés de façon continue), et les RP ne mesurent que l'un des deux statuts possibles. Il y a tout lieu de penser que lorsque un écolier est scolarisé à temps complet, qu'il travaille ou non par ailleurs, il est déclaré comme écolier. Un écolier qui n'est scolarisé que de façon discontinue (ce qui n'est possible que dans le primaire, 15 jours d'absence sans motif entrainnant le renvoi d'un écolier du préparatoire) sera probablement déclaré comme tel s'il ne travaille pas ; s'il travaille, il est impossible de savoir sous quel statut le chef de famille choisira de le déclarer.

L'on pourrait bien sûr imaginer un cas idéal où, d'une part, la statistique scolaire n'enregistrerait que les véritables scolarisés et où, d'autre part, les RP enregistreraient comme actifs tout enfant travaillant de façon significative. La somme de ces deux effectifs dépasserait Ceux de la classe d'âge correspondante d'un chiffre qui donnerait le nombre d'enfants à la fois travailleurs et scolarisés. Mais attendre un résultat de cette méthode serait ne pas tenir compte d'un quatrième cas de figure.

Quatrième cas de figure

II concerne les enfants qui ne sont ni scolarisés ni travailleurs. Dans cette catégorie, on peut en outre trouver des jeunes filles non scolarisées qui s'adonnent, dans le cadre de l'unité familiale, à des travaux domestiques et/ou ménagers. Ainsi, les tâches comme la garde de frères et sœurs plus jeunes, le ménage, etc., ne sont pas considérées comme du «travail » selon la définition économique habituellement retenue, même si, souvent, l'exercice de ces activités permet à la mère d'avoir un emploi rémunéré.

Égypte/Monde arabe, 18-19| 1994 

population inactive, une catégorie intitulée "travail domestique", à laquelle ne correspondent que des effectifs féminins dont une proportion importante de moins de 15 ans; Le travail domestique n'est considéré comme une véritable activité ni par les enquêtes, ni par le statisticien. Par ailleurs, le fait que ce soit le chef de famille qui réponde à ce type de questionnaire et que ce dernier soit en général le père, contribue à la sous-déclaration de l'éventuel travail productif de sa fille de moins de 15 ans, surtout quand ce travail s'effectue à la maison; de ce fait, ce qui est en réalité un travail productif (à temps partiel, mêlé à un travail ménager, etc.) se retrouve enregistré comme « travail domestique ».

On peut sans exagération affirmer que dans ce cas, la nomenclature statistique reflète une vision masculine du monde social. Et ce n'est certainement pas par hasard si, à côté de la catégorie "travail domestique ", réservée uniquement au genre féminin, existe une catégorie intitulée « ne désirant pas travailler » (catégorie distincte des chômeurs) et qui concerne, cette fois-ci. presque uniquement des jeunes garçons. Quoiqu'il en soit de ces biais idéologiques introduits dans la vision statistique du monde social, il n'en reste pas moins que les RP égyptiens permettent de comptabiliser les 6-14 ans qui ne sont ni scolarisés, ni travailleurs (avec, pour les filles, l'ambiguïté soulignée plus haut).

Ainsi, parmi les quatre cas de figure qui viennent d'être présentés, la statistique fournit des données chiffrées sur les effectifs scolaires, les effectifs d'actifs et les effectifs d'inactifs non scolarisés. Ceci n'est le cas qu'en ce qui concerne les résultats des derniers recensements. Dans ce qui suit, l'on examinera principalement l'évolution des effectifs d'enfants de 6-14 ans actifs et ce, depuis 1917. Ce n'est que pour la période la plus récente - les sources le permettant alors - qu'ils seront rapprochés des effectifs scolaires et des deux autres catégories définies plus haut.

Quel éclairage les statistiques de l'emploi nous donnent-elles sur l'évolution du travail des enfants en Égypte?

En Égypte, la proportion des 6-14 ans dans la population totale reste quasi constante entre 1917 et 1986 : elle oscille entre 24,3 et $26,9 \%$, la proportion de garçons dans la population masculine étant toujours supérieure d'un point et demi à celle des filles dans la population féminine. Durant cette période, les effectifs de 6-14 ans ont presque quadruplé, passant de 3,3 millions en 1917 à environ 11,9 millions au recensement de 1986 (de 12,7 à 48,3 millions en ce qui concerne la population totale). Il existe deux sources statistiques officielles qui saisissent l'activité économique des enfants. Ce sont les recensements décennaux de population (RP), d'une part, et les enquêtes emploi (EE), d'autre part, réalisées par les différents organismes officiels de la statistique qui se sont succédé en Égypte - le CAPMAS ${ }^{4}$ depuis 1964. Ces deux sources mesurent différemment le niveau de l'emploi en Égypte ; en effet, les recensements de population, exhaustifs, ne donnent que des niveaux généraux de l'emploi à des périodes très espacées les unes des autres, soit tous les 10 ans. A l'inverse, les enquêtes emploi, annuelles, sont supposées suivre les évolutions récentes du " marché de l'emploi. »

La première source de données sur l'emploi (RP) nous permettra de retracer l'évolution du travail des enfants de 1917 à 1976. Nous utiliserons pour la période suivante, allant de 1977 à 1988, la deuxième source (EE). La volonté de l'Etat de collecter exhaustivement, à travers les RP, des informations sur de nombreux sujets a en général pour conséquence que les questions posées sont à la fois simples et parfois succinctes. Les RP égyptiens offrent une bonne description des structures démographiques et

Égypte/Monde arabe, 18-19 | 1994 
sociales du pays et constituent un indispensable outil d'observation des grandes tendances de la société égyptienne. Par ailleurs, le recensement n'est pas seulement une source d'informations statistiques mais aussi une base d'échantillonnage pour de nombreuses enquêtes par échantillon qui viennent répondre à des interrogations auxquelles le recensement ne peut répondre. Tel est le cas, notamment, des enquêtes annuelles par sondage utilisées pour collecter des données sur l'emploi; la base d'échantillonnage censitaire donne à ces enquêtes une grande fiabilité. S'agissant de l'emploi, ces deux sources statistiques présentent le grand avantage de saisir la population active dès l'âge de 6 ans et distinguent deux classes d'âge (5 puis 6-9 ans et 10-14 ans pour les RP, et 6-11 ans et 12-14 ans pour les EE) ce qui permet de calculer des taux d'activité par groupes d'âge et donc de retracer l'évolution par âge du travail des enfants.

Après avoir passé en revue les problèmes de mesure posés par ces sources, on dégagera les grandes tendances de l'évolution, de 1917 à nos jours, du travail des 6-14 ans au niveau national. Puis on abordera les spécificités de cette évolution par groupes d'âge et par sexe. Enfin, les caractéristiques socio-économiques de l'emploi des enfants (branches d'activité, statut dans la profession, stabilité dans l'emploi, etc.) seront examinées à partir des informations données par l'EE de 1988 (EE 88), plus complètes et plus riches que celtes fournies par les RP et autres EE.

Problèmes posés par les sources statistiques

Dans tous les recensements égyptiens - depuis le quatrième, datant de 1917 (RP 17) -, les individus auxquels est attribuée l'appartenance à une branche d'activité constituent ce que nous appellerions aujourd'hui des "actifs occupés». Ce sont, parmi ces individus, les 5/6-14 ans qui ont été systématiquement retenus pour l'analyse du travail des enfants. Il est à noter que des enfants chômeurs sont mentionnés dans certains recensements; lorsque c'est le cas, ils font partie de la population active - où ils forment alors une fraction de celle-ci dite " active non occupée » - et dans d'autres, ils sont, comme la totalité des chômeurs, exclus de la population active. Dans tous les cas, les enfants chômeurs n'ont pas été retenus dans l'analyse. L'on ne prendra en compte que des 5/6-14 ans actifs occupés, toujours référés à une branche d'activité économique, définie ou inconnue. S'agissant de l'âge initial, les quatre premiers recensements (de 1917 à 1947) ont fixé 5 ans, les suivants 6 ans. Les effectifs absolus d'enfants actifs occupés n'ont pas été ajustés pour tenir compte de cette variation. Ils ne sont donc pas comparables dans le temps. Par contre, les taux nets d'activité (TA) à 5-14 ans aux RP de 1917 à 1947, et à 6-14 ans ensuite permettent de comparer les actifs occupés à la population totale du même âge.

30 Pour ce qui est de l'âge initial auquel la statistique saisit le travail, il est remarquable que, malgré des préoccupations précoces en matière de protection de l'enfance (dès 1900) qui se traduiront, à partir de 1932, par des lois fixant un âge minimal d'admission au travail (9 ans), l'appareil statistique se soit donné les moyens de repérer des pratiques réelles, y compris illégales.

31 Si les RP égyptiens saisissaient, au début du siècle, l'activité enfantine dès l'âge de cinq ans, c'est sans nul doute qu'à l'époque, les enfants tenaient une place importante dans la population active ; la tradition de tenir une statistique de l'activité précoce s'étant ainsi établie, elle sera conservée, ce qui fait que, de nos jours, l'âge initial, retenu pour mesurer le travail est extrêmement bas en comparaison de celui qui est pris en compte dans les autres pays en développement, de tradition statistique en général plus récente, 
dans lesquels l'âge initial de saisie statistique du travail se confond avec l'âge minimal officiel d'entrée dans la vie active, qui tourne généralement autour de 15 ans $^{6}$. Cette saisie du travail à un âge précoce est bien sûr une chance pour l'historien et le sociologue.

De manière générale, l'analyse des séries temporelles requiert certaines précautions. En particulier, les recensements modernes n'enregistrent que l'activité exercée la veille de la date officielle du RP - c'est le cas en Égypte depuis 1960 - ce qui constitue une période de référence extrêmement brève. Aucune autre difficulté majeure ne s'oppose à l'interprétation sur l'activité. Par contre, la mesure de la population active occupée présente deux difficultés : l'activité des garçons aux RP 17 et RP 47 et celle des filles au seul RP 17.

La catégorie « activité mal définie » et le calcul des TA des garçons aux RP 17 et RP 47

Pour mesurer la population active en l'absence de sous-totaux d'actifs et d'inactifs, il faut recourir aux nomenclatures dites de groups of occupations, qui correspondent à ce que nous appellerions maintenant «branche d'activité »; or cette dernière inclut une catégorie particulière d'individus actifs à la date de référence, catégorie nommée dans le RP 17 general designation without indication of a determined occupation (désignation générale de la branche d'activité sans précision exacte de cette dernière) et, dans les trois suivants, ill-defined occupation ${ }^{7}$ (activité mal définie). Aux RP 17 et RP 47, les effectifs répondant à cette catégorie sont exceptionnellement nombreux (tableau 1). En effet, selon Nassef, «les effectifs de ce groupe étaient beaucoup plus importants, en nombre et en proportion, aux RP 17 et RP 47 que dans les autres recensements. Au RP 47 , les individus de ce groupe n'avaient fait mention ni de leur branche d'activité, ni même de leur statut dans la profession. $»^{8}$

Tableau 1 : L'activité mal définie dans les recensements de population

\begin{tabular}{|l|l|l|l|l|l|l|}
\hline & Sexe & 1917 & 1927 & 1937 & 1947 & 1960 \\
\hline \hline Moins de 15 ans & Garçon & 95.214 & 614 & 42 & 231.343 & 6.006 \\
\hline \hline & Fille & 4.047 & 93 & 2.610 & 6.952 & 2.618 \\
\hline \hline Population active & Homme & 347.517 & 10.877 & 616 & 344.145 & 43.247 \\
\hline \hline totale & Total. & 99.261 & 707 & 2.652 & 238.295 & 8.624 \\
\hline \hline (5 ans et plus) & Total & 438.916 & 11.640 & 3.433 & 353.934 & 43.914 \\
\hline
\end{tabular}

Source : recensements de population.

On pourrait voir, dans le gonflement de la rubrique «activité mal définie », une volonté de rigueur des recenseurs; dans ce cas, ce phénomène s'exercerait aux dépens des activités bien définies, de sorte que la progression générale de la population active ne s'en verrait pas modifiée, la proportion actifs/non actifs évoluant régulièrement. Or ce 
n'est pas le cas : le surnombre d'«activités mal définies » entraîne, aux RP 17 et RP 47, un surcroît de l'activité globale.

La catégorie "activité mal définie » regroupe 439.000 actifs au RP 17 (soit 8,5\% de la population active de plus de 5 ans) et 354.000 au RP 47 (soit $5 \%$ ). Parmi ceux-ci, les 5-14 ans comptent pour $22,6 \%$ au RP 17 (ils sont 99.000) et pour 67,3\% au RP 47 (ils sont 238.000). Comme on le constate, les 5-14 ans sont surreprésentés dans cette catégorie. Au RP 17, les 5-14 ans appartenant à cette dernière sont en fait constitués, dans leur quasi-totalité, de garçons de 10-14.ans (soit $95 \%$ ) ; au RP 47, ce sont les garçons des deux sous-classes d'âge qui constituent l'essentiel de cette catégorie (soit $97 \%$ ). Ainsi aux deux recensements, le gonflement de la catégorie "activité mal définie » ne concerne que les effectifs masculins. Il faut préciser que les effectifs de 5-14 ans correspondant à cette catégorie sont insignifiants dans les autres RP (1927.37 et 1960).

Dans l'analyse des grandes tendances de l'évolution des taux d'activité des enfants pour les deux sous-groupes d'âges distincts (i. e. de la mesure de la population active occupée), nous avons délibérément retenu, pour le calcul des taux d'activité, les seuls effectifs de population active appartenant aux branches bien définies; on ne tiendra donc pas compte de la catégorie " activité mal définie » en raison de la cohérence que cette exclusion permet de rétablir dans l'évolution des effectifs actifs ${ }^{9}$.

Le surenregistrement des filles au RP 17

Le deuxième problème concerne uniquement les filles de 5-14 ans au RP $17^{10}$. La mesure de l'activité des filles y a fait l'objet d'un traitement tout à fait particulier ; l'affectation automatique d'un grand nombre de femmes et de filles de plus de 8 ou 10 ans, selon les cas, à deux catégories d'activité données. En effet, les instructions aux agents recenseurs stipulaient, premièrement, que les femmes et les filles de plus de 10 ans appartenant à des familles de propriétaires fonciers possédant plus de 10 feddans, de même que celles qui appartiennent à des familles dont l'occupation du chef de ménage n'est pas mentionnée, exerçaient un «travail domestique » qui, lors de ce recensement, était considéré comme une véritable activité économique : 254.000 filles de 10 à 14 ans entraient dans celte catégorie; deuxièmement, que les femmes et les enfants de plus de 8 ans, ne fréquentant pas l'école et qui appartenaient à des familles d'exploitants agricoles cultivant moins de 10 feddans seraient considérés comme "travailleurs agricoles employés dans l'exploitation familiale »: 256.000 filles de 10 à 14 ans ont fait l'objet de cette affectation. Le fait que les RP suivants n'aient pas effectué ce type d'affectation automatique, que le travail domestique n'ait plus été considéré comme une véritable activité économique et que la catégorie "aide familiale agricole» féminine n'ait plus été retenue conduit à éliminer, à des fins de comparabilité, les 500.000 filles de 10 à 14 ans comptées dans ces deux catégories et dont la prise en compte conduisait à un taux d'activité record pour cette classe d'âge, à savoir $79 \%$ (contre 15,5\% après déduction de ces 500.000 filles).

38 S'agissant des 256.000 travailleuses agricoles familiales ayant entre 10 et 14 ans, il est à remarquer que, compte tenu de l'importance de l'emploi, à cette époque, d'une maind'œuvre saisonnière agricole - pour la récolte du coton en particulier - ce chiffre n'est nullement irréaliste.

39 Se pose donc bien le problème de la définition de l'activité (périodicité et durée du travail). En effet, si le travail dans l'agriculture avait de fortes chances d'avoir pour les garçons un caractère permanent ou en tout cas régulier, pour les filles, la durée de leur participation aux travaux agricoles ne dépassait pas quelques mois dans l'année. 
Le raccordement des données du RP 76 et de l'EE 77 recensements (la «nuit du recensement ") n'a jamais coïncidé avec celle des enquêtes emploi (dont la première remonte à 1957). Par ailleurs, les EE sont conçues différemment des RP, tant dans leurs méthodes et définitions que dans leur réalisation: elles visent en effet à répondre à des préoccupations plus spécifiques, en l'occurrence d'analyse du marché de l'emploi. Il était tentant de rapprocher les données de ces deux sources statistiques. Pour mener à bien cette comparaison, il convient de tenir compte de trois facteurs de « divergence » entre ces deux sources :

41 - traditionnellement, l'enquête emploi ne porte que sur la "population des ménages ordinaires » (opposée aux ménages collectifs : prisons, hôpitaux, etc) ;

42 - la définition de l'activité en général et du chômage en particulier n'est pas la même dans les deux sources. Si, dans les EE, la catégorie «chômeurs » est susceptible d'être appliquée à des individus ayant entre 12 et 64 ans, dans les RP, elle peut s'appliquer dès l'âge de 6 ans ;

43 - les questions posées sur l'activité, dans les deux sources, portent sur deux périodes de « référence » différentes : le jour précédant le recensement ou la semaine précédant l'enquête. Pour être considéré comme actif, il suffit donc d'avoir travaillé la veille du recensement ou la semaine ayant précédé l'EE. Par ailleurs, les dates de l'opération de l'EE 77 et du RP 76 étaient différentes: mai pour la première et novembre pour la seconde (généralement, ces dates sont choisies de manière à coïncider avec une période de creux des travaux agricoles, afin d'éviter les mouvements saisonniers de population).

44 A titre d'illustration, et ce, compte tenu de leur relative proximité dans le temps, la comparaison des données sur l'activité des enfants du RP 76 avec celles du l'EE 77 donne des résultats satisfaisants. Le rapprochement de ces deux sources statistiques révèle que d'après les déclarations enregistrées au RP 76 , le volume de la population active occupée de 6-14 ans était estimé à environ 992.000, ce qui représente, par rapport à la classe d'âge correspondante, un taux d'activité global de $12 \%$ et que, de son côté, l'évaluation plus stricte qu'autorisait l'EE 77 donnait un effectif très peu inférieur de 970.000, correspondant à un taux d'activité global de $11,5 \%$. Si l'on considère les taux masculins à 6-11 ans et à 12-14.ans selon l'EE 77, ils sont légèrement inférieurs à ceux enregistrés au RP 76, soit respectivement de $12,6 \%$ et $27 \%$ à l'EE contre $15,5 \%$ et $29 \%$ au RP. L'application dans les EE de critères plus exigeants quant à la mesure et à la définition de l'activité pourrait expliquer la légère différence, moins de 22.000 du volume global des 6-14 ans actifs à l'EE 77. En revanche, les TA féminins à 6-11 ans donnés par le RP 76 sont supérieurs à ceux donnés par l'EE 77: soit respectivement $2,2 \%$ contre 5,4 \%. Quant aux TA à $12-14$ ans, ils avoisinent $3 \%$ dans les deux sources.

Evolution de l'effectif des 6-14 ans au travail (1917-1988)

Même si l'analyse des variations intercensitaires de l'effectif des 6-14 ans au travail est un exercice difficile, en raison de la variabilité des concepts, des méthodes et des nomenclatures présidant à la définition et à la mesure de ce travail, la mise en série, chronologique des effectifs est: indispensable pour mener une analyse dynamique des taux d'activité de cette classe d'âge sur la longue période ${ }^{11}$. Cette analyse permettra seule, même si ses résultats ne mettent à Jour que des tendances, de donner du phénomène une image approchant l'«objectivité », face aux représentations

Égypte/Monde arabe, 18-19 | 1994 
contradictoires que s'en fait l'opinion ${ }^{12}$. Or l'Égypte est un des rares pays en développement qui dispose de séries statistiques anciennes en général, et sur le travail des moins de 15 ans en particulier ${ }^{13}$.

L'évolution du TA (taux d'activité) global à 6-14 ans en Égypte depuis 1917 permet de repérer trois périodes bien distinctes (fig. 1) :

-1917-1937 : quasi-plafonnement du TA à un niveau élevé (20 \%) ;

- 1937-1976 : déclin régulier de l'activité ;

- 1976-1988 : stabilisation du TA (12\%).

Si l'examen diachronique du travail des enfants en Égypte, au travers de l'évolution des taux d'activité globaux (c'est-à-dire sexes et âges confondus) permet de dégager de grandes tendances, en revanche seul un examen détaillé par sexe et par sous-groupes d'âge (d'abord 6-9 ans et 10-14 ans pour les RP puis 6-11 ans et 12-14 ans pour les EE) nous permettra de nuancer nos impressions premières, qui peuvent occulter des évolutions différentielles, selon le sexe et l'âge, de l'activité enfantine au cours de ces 70 ans.

Fig. 1 : Evolution du taux d'activité (garçons et filles) des 6-14 ans en Égypte, 1917-1988

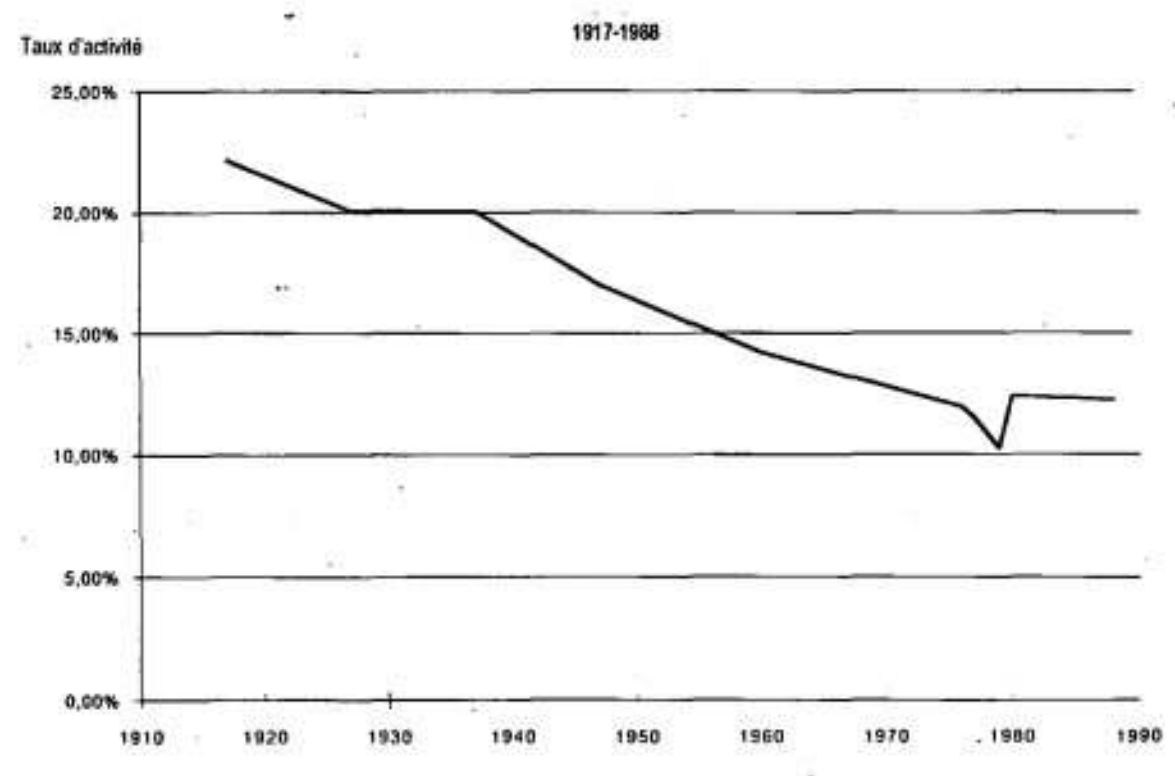

Les trois étapes dégagées dans l'évolution des taux d'activité globaux des 5/6-14 ans sur 70 ans se retrouvent-elles dans l'évolution des TA de chaque sexe de la même tranche d'âge ? Un coup d'œil sur la figure 2 permet de constater d'emblée que ce n'est pas le cas. Dans la mesure où, jusqu'au milieu des années 70, le nombre de filles de 5/6-14 ans travaillant reste très inférieur à celui des garçons (les filles actives représentent, selon les recensements, un effectif 3 à 8 fois moins nombreux que celui des garçons), la forme de la courbe représentant l'évolution des TA globaux s'explique en très grande partie par l'évolution des TA masculins. L'on retrouve bien, dans la courbe qui figure cette dernière, le quasi-plateau élevé des années 1917-37, puis une pente plus raide et plus courte (de 1937 à 1960), suivie non plus d'un palier, mais d'une pente douce qui s'infléchit nettement vers la fin de la période analysée (année 1988). 

dans les niveaux. En 1917-37, ce sont autour de $32 \%$ des garçons de 5-14 ans qui travaillaient, selon les RP ; ils n'étaient plus que $20 \%$ en 1960 et environ $13 \%$ en 1988. S'agissant du TA féminin des 5/6-14 ans, toujours très inférieur à celui des garçons, il connaît, durant toute la période et jusqu'à la fin des années 70, une lente diminution: parti de $8,3 \%$ en 1917, il descend à $3 \%$ vers l'extrême fin des années 70 . Il était, à la première date, 4,5 fois moins élevé que celui des garçons ; il en représente le cinquième en 1979, ce qui signifie qu'il a décliné un peu plus vite que celui des garçons durant les 60 ans qui séparent ces deux dates; cependant, ce déclin a été très régulier, contrairement à celui du TA masculin.

Ce qui différencie cependant bien davantage l'évolution des TA selon le sexe durant la période 1917-88, c'est la remontée sensible du TA féminin durant les 10 dernières années de la période, où il passe de $3 \%$ à $11,6 \%$, rejoignant presque celui des garçons qui a connu durant les mêmes années, on l'a vu, une évolution inverse. Le rythme et la tendance des variations des TA selon le sexe, non moins que leur niveau à chaque date de recensement ou d'enquête, tout distingue l'évolution du travail des filles et des garçons de 5/6-14 ans durant les 70 dernières années, le trait le plus remarquable étant sans doute le presque quadruplement du TA féminin de 1979 à 1988, qui correspond à un quintuplement des effectifs (compte tenu de l'augmentation de la population de la tranche d'âge durant ces dix années) ${ }^{14}$. Cette brusque remontée de l'activité féminine paraît à bien des égards suspecte. Loin de constituer un réel changement social, il semblerait que cette rupture de pente soit davantage le résultat d'un changement - de définition - qui s'est opéré dans l'observation statistique du phénomène. On peut donc penser que toute la période précédente (avant les années 80) a été caractérisée par un sous-enregistrement généralisé du niveau d'activité des filles et ce, du fait du caractère invisible des emplois occupés par ces dernières.

Fig. 2 : Evolution du taux d'activité des 6-14 ans en Égypte, 1917-1988

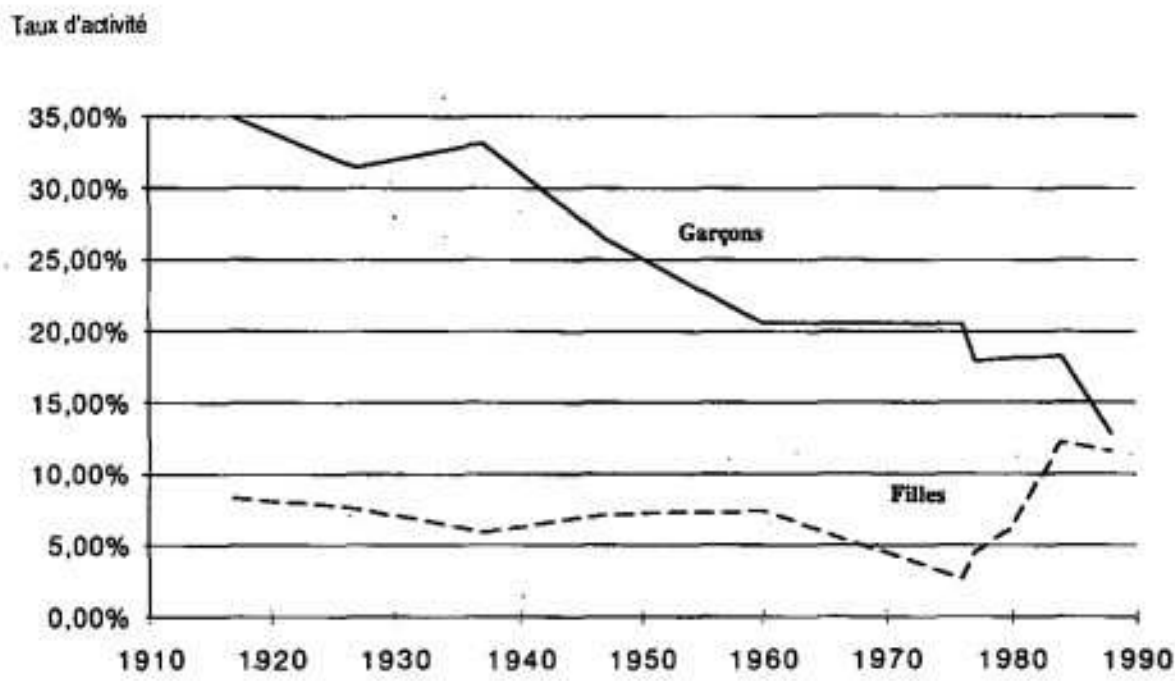

Une désagrégation des taux d'activité par tranche d'âge permet-elle une meilleure perception de l'évolution de ces derniers? Pour ce qui est du rythme et des tendances 
d'évolution des TA des garçons et des filles de 10/12-14 ans (fig. 3), ils respectent la périodisation dessinée par les TA à 6-14 ans, pour chaque sexe. L'on retrouve bien trois périodes pour les garçons de 10/12-14 ans, avec un plafond initial entre 1917 et 1937, une baisse rapide de 1937 à 1960 et une légère érosion de 1960 à 1988. L'on retrouve également la longue période de déclin du TA féminin de 1917 à 1979, suivie d'une remontée dans les 10 dernières années (ces ressemblances s'expliquant là encore en grande partie par le poids des 10/12-14 ans dans la population totale des actifs de 5/6-14 ans).

S'agissant maintenant des enfants de 5/6-9/11 ans (fig. 4), on retrouve une lente érosion de l'activité des filles entre 1917 et 1976, suivie d'une remontée, mais la périodisation de l'évolution du TA des garçons est plus délicate à esquisser, vu son extrême variabilité selon les recensements et les enquêtes : si on laisse de coté le taux extrêmement bas obtenu au RP 37, l'on peut admettre l'existence d'un quasi-plateau entre 1917 et 1976, suivi d'un déclin de 1976 à 1986. Il est remarquable que s'agissant des 5/6-9/11 ans comme des 10/12-14 ans, deux tendances inverses caractérisent, dans les 10 dernières années, l'évolution des TA selon le sexe, aboutissant à une quasi convergence des TA des deux sexes à un niveau de $12 \%$ pour l'ensemble des 6-14 ans, à 20-24\% pour les 12-14 ans et à 7-7,5\% pour les 6-11 ans.

Les régularités constatées dans la périodisation des évolutions des TA masculins, d'une part (entre 5/6-9/11 ans et 10/12-14 ans, avec trois phases : plafond, baisse brutale, lente érosion), et des TA féminins, d'autre part (avec deux phases: lente érosion 1917-1976/79 et remontée rapide 1979-88), ne doivent pas dissimuler la très forte disparité de leurs niveaux, à une même date, selon le sexe et l'âge (la dernière décennie étant exclue) : pour les garçons, la première période oppose un TA des 10-14 ans de 55 $\%$ à un TA des 5-9 ans de $13 \%$ (soit un rapport de 1 à 4); en début de troisième période (1960), le TA des premiers est de $28 \%$, ce qui correspond à la moitié de ce qu'il était durant la première période, tandis que le TA des second se maintient à $13 \%$. Le rapport de ces deux taux masculins n'est plus que de 1 à 2 en 1960, mais il s'élève de nouveau légèrement en fin de troisième période (de 1 à 3 en 1988 : le TA des 12-14 ans est de 24 $\%$ et celui des 6-11 ans de 7,5\%). Cependant, les TA des deux classes d'âge masculines prises en compte n'ont diminué que de moitié en 70 ans (un peu plus pour les 10/12-14 ans et un peu moins pour les 5/6-9/11 ans). 
Fig. 3 : Evolution du taux d'activité des 10-14 ans en Égypte, 1917-1988

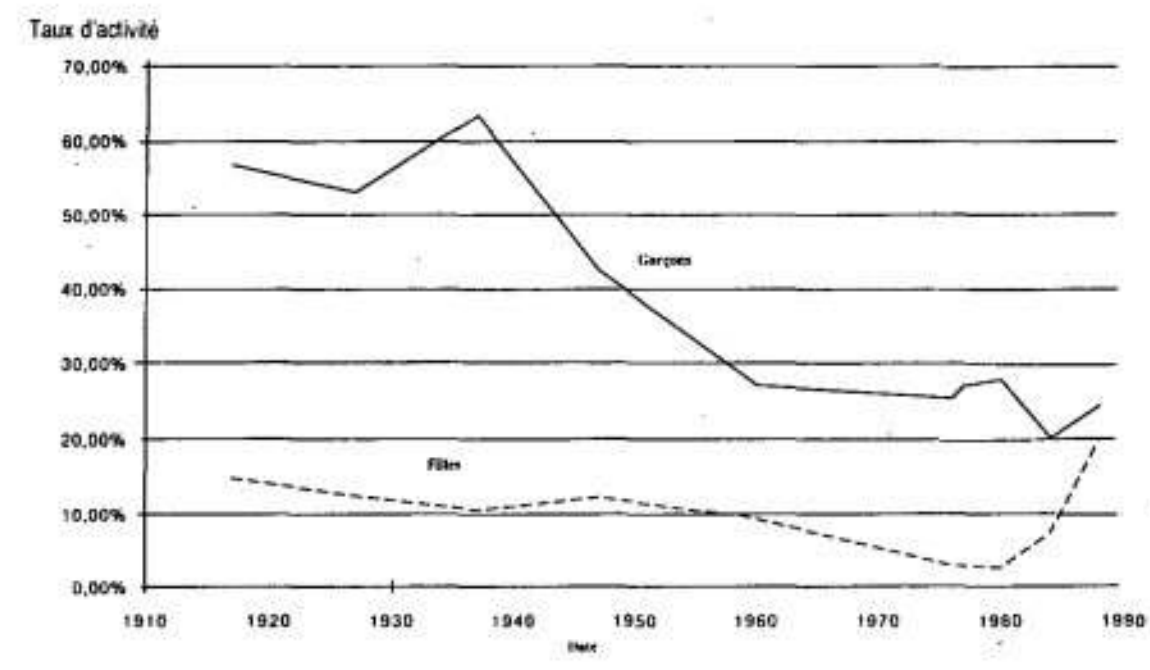

Fig. 4 : Evolution du taux d'activité des 6-9 ans en Égypte, 1917-1988

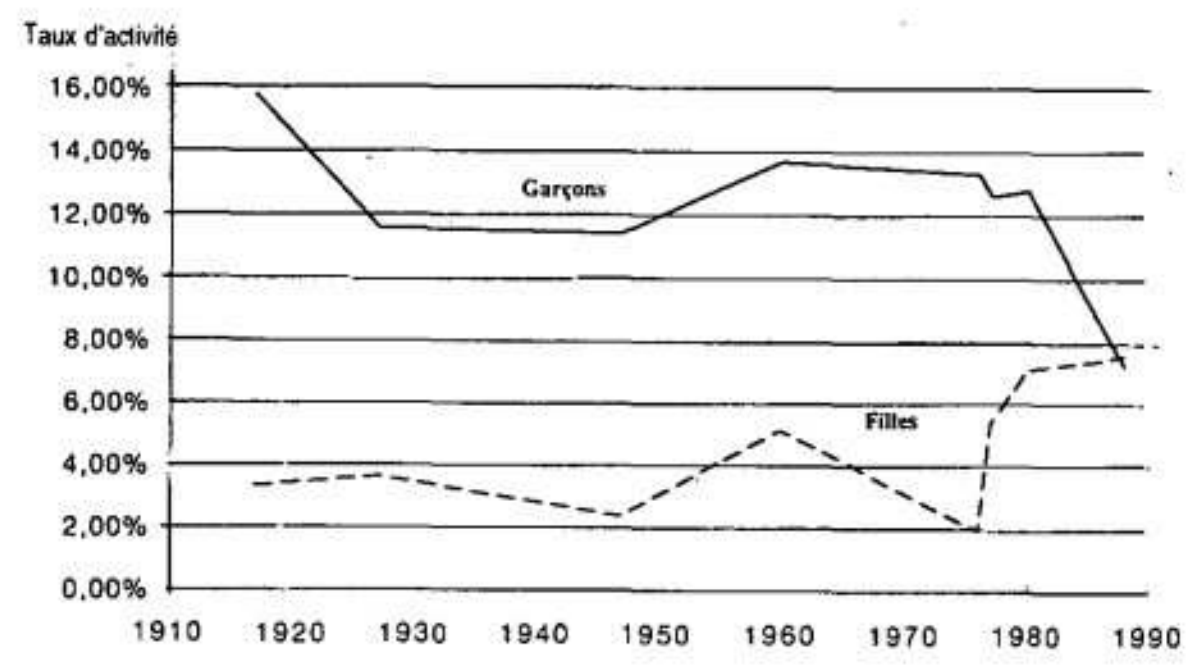

L'activité féminine est à chaque âge inférieure à l'activité masculine (l'année 1988 étant exclue, qui marque, on l'a vu, la convergence de ces taux). La première phase voit passer le TA des filles de 10/12-14 ans de $15 \%$ à $2 \%$, la seconde le voit remonter à $20 \%$, niveau atteint en 1986, alors que les TA des 5/6-9/11 ans ne dépassent pas 5,5 \% entre 1917 et 1977, pour passer à 7,5 \% en fin de deuxième phase. En 1988, donc, l'âge apparaît comme tout à fait discriminant quant au TA, alors que le sexe ne l'est plus du tout. Sur l'ensemble de la période considérée (1917-88), par contre, le sexe et l'âge intervenaient tous deux de façon significative dans la différenciation des niveaux d'activité.

L'analyse en termes de taux d'activité ne rend compte que d'une partie du phénomène analysé, puisqu'elle réfère un ensemble d'actifs à la population totale de la classe d'âge correspondante. Il peut être également intéressant de prendre cette population active 
en elle-même et d'analyser l'évolution de ses effectifs et de sa composition interne. Pour ce qui est des effectifs, il serait hasardeux de prendre en compte séparément les deux sous-groupes d'âge distingués, dans la mesure où les limites d'âge de ces derniers changent deux fois. L'on sait déjà, par la baisse du TA global des 5/6-14 ans, que les effectifs d'enfants actifs n'ont pas crû au rythme de la population totale de la classe d'âge correspondante (tableau 2 p. suiv.). Ces effectifs d'actifs ont doublé en 70 ans, passant de 0,7 million à 1,4 million. Les effectifs de garçons ne connaissent qu'une augmentation de $50 \%$ entre 1917 et 1984 (ils retombent à 750.000 en 1988) alors que, les effectifs féminins quintuplent durant la même période (ils passent de 130.000 à 650.000). Ce rapide survol des effectifs absolus permet de rendre compte du fait que si les TA des enfants de 6-14 ans ont diminué durant la période de référence, le travail des enfants pris dans son importance démographique absolue est plus massif que jamais. Bien entendu, les actions qui viseraient à le combattre, si elles peuvent être encouragées par la baisse relative du phénomène, doivent déployer des moyens correspondant à son ampleur inédite.

Travail et scolarisation d'après l'enquête par sondage d'octobre 1988

Deux mesures utiles de la population active sont, d'une part, la population habituellement active mesurée en fonction d'une longue période de référence comme l'année, et la population active du moment, appelée encore main-d'œuvre, et mesurée par rapport à une courte période de référence: un jour ou une semaine. La date de référence de l'enquête par sondage sur la population active égyptienne a été fixée au 31 octobre $1988^{15}$, et selon cette même enquête, la population égyptienne totale a été estimée à environ 51 millions de personnes. Les moins de 15 ans représentaient $41 \%$ de la population totale; le groupe d'âge 6-14 ans s'élevait à 11,33 millions dont 7,6 entre 6 et 11 ans.

Tableau 2 : Evolution du taux d'activité des 6-14 ans en Égypte, 1917-1988.

\begin{tabular}{|c|c|c|c|c|c|c|c|c|c|c|c|c|c|c|c|}
\hline \multirow{3}{*}{ Annose } & \multirow{3}{*}{$s^{*}$} & \multicolumn{6}{|c|}{ Population adtioe (\$.14 ans; } & \multicolumn{4}{|c|}{ Population adive $(6-9$ ans\}) } & \multicolumn{4}{|c|}{ Popultion activo (10-14 ans) } \\
\hline & & \multicolumn{3}{|c|}{ Elloctils } & \multicolumn{3}{|c|}{$T A^{*}$} & \multicolumn{2}{|c|}{ Eloctits } & \multicolumn{2}{|c|}{$T^{* H}$} & \multicolumn{2}{|c|}{ Eftectits } & \multicolumn{2}{|c|}{ TA* } \\
\hline & & Garpens & Filles & Total & G & $F$ & Total & Gavpons & Fillins & 6 & F & Garçons. & Filles & 6 & F \\
\hline 1917 & $R^{P}$ & 583.575 & 130,170 & 723745 & $35 \%$ & $8 \%$ & $22 \%$ & 142.644 & $30 \mathrm{~cm}$ & $16 \%$ & $3 \%$ & 450.931 & 100096 & $37 \%$ & $15 \%$ \\
\hline 1927 & AP & 565.536 & 124.317 & 609.653 & $31 \%$ & $6 \%$ & $20 \%$ & 108.426 & 30.687 & $12 \%$ & $4 \%$ & 457,110 & 90.600 & $50 \%$ & $13 \%$ \\
\hline 1937 & AP & 702.644 & 117034 & 825.578 & $33 \%$ & 68 & $20 \pi$ & 55.04 & 23.645 & $5 \%$ & $2 \%$ & 653600 & 93.390 & 608 & $11 \%$ \\
\hline 1947 & $A P$ & 202.406 & 161221 & 763.707 & $26 \%$ & $7 \%$ & $17 \%$ & 137.962 & 28.428 & $11 \%$ & $2 \%$ & L44534 & 192.793 & $42 \%$ & $12 \%$ \\
\hline 1950 & $\mathrm{RP}$ & 65e.847 & 218,790 & 877645 & $21 \%$ & 75 & $14 \%$ & 212.942 & 74.650 & $14 \%$ & $5 \%$ & 445.905 & $-744,145$ & $27 \%$ & $9 \%$ \\
\hline 1976 & AP & 886.969 & 103.597 & 990566 & $21 \%$ & $3 \%$ & $12 \%$ & 238.765 & $31.46 \mathrm{~V}$ & $13 \%$ & $2 \%$ & 654204 & 72.130 & $25 \%$ & $3 \%$ \\
\hline $19 \pi 7$ & EMO & ress 300 & 181.400 & 969.700 & $18 \%$ & $5 \%$ & $12 \%$ & 352.500 & 139.400 & $13 \%$ & $5 \%$ & 425.800 & 42.000 & $27 \%$ & $3 \%$ \\
\hline 1980 & EMO & 842.400 & 259.900 & $1.100,300$ & $18 \%$ & $6 \%$ & $12 \%$ & 300.600 & 218,800 & $13 \%$ & $7 x$ & $4 S B 800$ & 41.100 & $28 \times$ & $3 \%$ \\
\hline 1984 & EMO & 911300 & 561.300 & 1.872 .500 & $18 \%$ & $12 \%$ & $15 \%$ & 568,300 & 446500 & $17 \%$ & $15 \%$ & 343000 & 115.300 & $20 \%$ & $8 \%$ \\
\hline 1968 & ENo & 746000 & \$4t.000 & 1.387 .000 & $13 \%$ & $12 \%$ & $12 \%$ & 281.000 & 200.000 & $7 \%$ & $8 \%$ & 465.000 & $36 \div, 000$ & $24 \%$ & $20 \%$ \\
\hline
\end{tabular}

* Source : CAPMAS : recensements de la population égyptienne (RP) et enquêtes main-d'œuvre sur échantillon (EMO).

** TA: taux d'activité.

60 Compte tenu des différences majeures qui ont présidé tant à sa conception qu'à sa réalisation, les résultats de l'enquête-emploi d'octobre 1988 ne sont, sur certains points, comparables ni aux RP, ni même aux autres EE la précédant ou lui succédant ${ }^{16}$. En effet, cette enquête a été modifiée de manière à élargir et à approfondir la mesure de l'emploi; meilleure répartition de l'échantillonnage sur l'ensemble du pays ${ }^{17}$, définition et mesure précises de l'emploi et du chômage déclaré grâce à un jeu de 
questions de contrôle, essais de mesure du sous-emploi, utilisation de trois périodes de référence pour mesurer les caractéristiques de l'emploi (une semaine, trois mois et un an), contrôle de qualité élevé à tous les stades de l'enquête ${ }^{18}$.

Si le "professionnalisme" avec lequel cette enquête a été menée augure bien de la fiabilité de ses résultats, le caractère unique de cette enquête fait qu'elle mérite un examen attentif. Pour la première fois dans l'histoire de la statistique égyptienne, grâce à cette enquête, un traitement particulier concernant l'activité des enfants a été réalisé. Pour ce faire, un questionnaire spécifique (module $\mathrm{n}^{\circ} 5$ ) portant sur «les conditions de travail des 6-14 ans » a été conçu, et les questions de ce module ont été adressées directement aux personnes concernées, c'est-à-dire à ceux ou celles qui ont exercé une activité économique au cours des trois périodes de référence prises en compte. Ont cependant été délibérément exclus des enquêtes les moins de 15 ans dont le statut dans la profession est « travailleur familial non rémunéré ».

Aspect démographique du travail des 6-14 ansLe volume global de la population active des 6-14 ans

D'après les résultats de l'enquête, la population active des 6-14 ans est estimée à 1,387 million d'enfants au cours de la courte période de référence ${ }^{19}$, c'est-à-dire la semaine précédant l'enquête, ce qui correspond, on l'a vu, à un taux d'activité de 12,25\%. La proportion des actifs de 6-14 ans dans la population active totale âgée de 6 ans et plus (soit 18,636 millions) était de 7,5\%. En dépit de la scolarité obligatoire des enfants appartenant à cette classe d'âge (loi 139, 1981), le taux d'activité global des 6-11 ans par rapport à la classe d'âge correspondante était de 7,4\%, soit un nombre total d'enfants de 561.000 , contre $22,3 \%$ chez les $12-14$ ans, soit 826.000 au moment de l'enquête. Si les TA à 6-11 ans sont pratiquement identiques chez les garçons et les filles (7,2 \% et 7,5\%) à 12-14 ans, le TA des garçons est supérieur de 4 points à celui des filles, soit respectivement $24,4 \%$ et $20 \%$.

63 Si l'on admet que l'intensité de l'activité des enfants augmente avec la durée de la période de référence et que l'on considère maintenant la période de référence d'un an, l'on peut s'attendre à une augmentation du TA, ce qui est le cas d'après l'enquête 1988; 2,2 millions d'enfants de 6-14 ans ont en effet exercé une activité économique au cours de l'année qui a précédé l'enquête, ce qui représente un taux d'activité de 19,4 \% (22,3 $\%$ chez les garçons contre 15,6 \% chez les filles). Les actifs de 6-14 ans représentent alors $11,8 \%$ de la population active totale âgée de 6 ans et plus. Malgré l'interdiction légale de travailler ou de suivre un apprentissage avant l'âge de 12 ans, ce sont près d'un million d'enfants (956.000) âgés de 6 à 11 ans qui exerçaient une activité économique au cours de la période de référence d'un an.

Les écarts de taux d'activité selon le milieu (urbain ou rural)

Les TA varient beaucoup selon la zone urbaine ou rurale de résidence. En effet, le TA des jeunes ruraux (17\%, soit 1,2 million) est nettement plus important que celui des urbains ( $4,25 \%$ soit 0,2 million) (note: la période de référence est de nouveau la semaine). Quelle que soit la tranche d'âge, les écarts de TA entre les sexes, selon la zone de résidence, présentent la même structure : en milieu rural, les TA des deux sexes sont pratiquement égaux; en zone urbaine, le TA des garçons est le double de celui des tilles.

65 Les TA des 6-14 ans sont respectivement de 16,2 \% et $17 \%$ chez les filles et les garçons ruraux contre $3 \%$ et $6,5 \%$ chez les urbains. Dans la classe d'âge 6-11 ans, les TA correspondants sont de $9,8 \%$ et $11,5 \%$ et de $1,5 \%$ et $3 \%$. Enfin, chez les $12-14$ ans, ils 
sont de $29 \%$ et $31 \%$ et de $6,2 \%$ et $13,6 \%$. Si le travail des enfants en ville concerne donc à $70 \%$ des garçons et à $30 \%$ des filles, c'est en raison de la nature même des emplois qui s'offrent en ville (travail salarié, apprenti dans un atelier sont des emplois masculins) et du fait qu'en ville, le travail des filles se déroule hors du cadre familial, ce qui constitue un frein à leur activité. Le trait important à souligner ici est qu'une fois de plus, la visibilité relative des emplois occupés par les garçons (comme c'est le cas des hommes par rapport aux femmes) fait qu'ils sont mieux appréhendés par la statistique que ceux des jeunes filles, qui occupent en général des emplois « invisibles » comme les travaux à façon, effectués à la maison et mêlés au travail domestique.

Enfin, les taux d'activité des 6-14 ans urbains sont plus élevés en Basse-Égypte (6,8 \%) et en Haute-Égypte $(4,8 \%)$ que dans le Grand-Caire $(3,7 \%)$ et à Alexandrie $(2,8 \%)$. L'activité des 6-14 ans est plus importante en Basse-Égypte rurale (18\%) qu'en HauteÉgypte rurale (14\%).

Caractéristiques de l'activité des enfantsBranche, statut, rythme et temps d'activité ; salaires

La description des caractéristiques de l'emploi des enfants, issue du module spécial "conditions de travail des 6-14 ans", permet pour la première fois de dresser un tableau général du phénomène (branche d'activité, statut dans la profession, secteur d'activité, stabilité dans l'emploi, chômage...).

A la question "Que font-ils?", il ressort, d'après les informations concernant la branche d'activité économique, que la grande majorité des moins de 15 ans travaille dans l'agriculture (1,019 million soit $75 \%$ de l'ensemble des actifs occupés); les filles actives y sont en proportion supérieure (53\%) aux garçons. Notons que, d'après la législation du travail, l'interdiction de travailler en dessous de 12 ans ne s'applique pas à l'agriculture. L'industrie, avec $9 \%$ des actifs occupés (soit 115.000), se range en deuxième position; c'est sans grande surprise que les garçons représentent $78 \%$ des actifs occupés à 6-14 ans dans ce secteur. Le commerce emploie $7 \%$ des actifs de 6-14 ans (soit 92.000) et les services 4,6 \% (soit 57.000) Dans le tertiaire, les garçons représentent $89 \%$ de l'emploi total des 6-14 ans : mécaniciens, repasseurs, etc.

Que ce soit à la campagne ou en ville, le secteur privé (entendons par là les exploitations agricoles familiales en zones rurales et les petites unités de production familiales et ateliers en zones urbaines, qui appartiennent pour la plupart au secteur informel) embauche la quasi-totalité des 6-14 ans actifs occupés. Les moyens de production utilisés dans ces secteurs d'activité sont faiblement capitalistiques et leur viabilité économique nécessite l'embauche d'une main-d'œuvre bon marché et docile, $C^{\prime}$ est également dans ces derniers que la réglementation du travail est la plus difficile à mettre en œuvre.

70 S'agissant du statut dans la profession, le travail des enfants s'exerce aux 3/4 (soit 995.000 enfants) sous le statut de «travailleur familial non rémunéré ». Parmi les «travailleurs familiaux non rémunérés », les deux sexes sont représentés à l'identique. Le statut de "salarié » arrive en deuxième position, avec 23 \% des 6-14 ans, soit 300.000 enfants dont $72 \%$ de garçons, et $75 \%$ des salariés ont entre 12 et 14 ans. Enfin, les « indépendants » ou "travailleurs à leur compte», en majorité des garçons, étaient 13.000 au moment de l'enquête.

71 Les emplois occupés par les moins de 15 ans peuvent par ailleurs revêtir des formes diverses : permanents, saisonniers, à la journée ou à la tâche. Par ailleurs, leur travail peut être « à plein temps » ou de quelques heures par jour et s'accomplir alors avant ou 
après l'école. La stabilité de l'emploi est certainement liée à sa régularité. Les résultats de cette enquête montrent que, sur l'ensemble des 6-14 ans actifs occupés interrogés, $76 \%$ avaient un emploi permanent (les effectifs des permanents se répartissant à égalité entre les 2 sexes). Les emplois occasionnels, c'est-à-dire à la tâche ou à la journée, concernent $19 \%$ des actifs occupés (soit 251.000 dont $65 \%$ de garçons, employés dans le secteur du bâtiment par exemple). Enfin, les emplois à caractère saisonnier représentent seulement $3,4 \%$ (soit 45.000 dont $70 \%$ de filles).

72 L'enquête indique une durée hebdomadaire de travail de 26 heures pour les 6-11 ans contre 34 heures pour les 12-14 ans (dans ce dernier groupe d'âge, la différence entre les sexes est significative ; 37 heures chez les garçons contre 29 heures chez les filles) alors que la moyenne, tous âges confondus, était de 39 heures en Égypte (avec une différence importante entre les hommes et les femmes, soit respectivement 45 et 30 heures). Si l'on considère la période de référence d'un an, les 6-11 ans actifs ont travaillé pendant 25 semaines (soit plus de la moitié de l'année) avec une légère différence entre les garçons et les filles (respectivement 23 et 26 semaines), et les 12-14 ans pendant 29 semaines. Notons que le nombre de semaines de travail pour l'Égypte, tous âges confondus, était en moyenne de 38 dans l'année : 40 pour les hommes et 35 pour les femmes.

73 L'activité scolaire est considérée comme un concurrent majeur du travail des enfants. Les garçons non scolarisés travaillent en moyenne chaque jour deux fois plus longtemps que les garçons scolarisés (6,2 heures contre 3,3), Sans que l'on puisse dire si c'est le fait d'être scolarisé qui limite le temps de travail à 3 heures en moyenne ou si c'est le fait de travailler 6 heures en moyenne par jour qui limite la scolarisation, la corrélation négative entre temps de travail et scolarisation semble claire. D'après l'enquête, il a été estimé que le salaire annuel moyen des enfants employés hors du cadre familial est de $277 \mathrm{LE}$, celui d'une jeune fille ne représentant que $78 \%$ de celui d'un garçon. Les enfants qui vont à l'école reçoivent moins de la moitié ( $46 \%$ ) de ce que reçoivent les enfants non scolarisés ${ }^{20}$. En général, les enfants travaillent parce qu'ils constituent une source de revenu non négligeable pour la famille. Les rentrées provenant du travail des enfants étaient d'environ $400 \mathrm{LE}$ en moyenne annuelle par famille, ce qui laisse penser qu'au sein d'une même famille bénéficiant des revenus rapportés par une main-d'œuvre enfantine, on trouve en moyenne 1,4 enfant actif. Ce montant de 400 LE représente $26 \%$ des revenus moyens de la famille. Ce qui signifie que la contribution financière de l'enfant qui travaille en dehors du cadre familial représente environ le quart des revenus du ménage. Les salaires des enfants actifs dépassent les $3 / 4$ des revenus du ménage dans plus de $10 \%$ des ménages ayant un ou plusieurs enfants travaillant en dehors du cadre familial. Par ailleurs, le quart des enfants travailleurs disent pouvoir disposer de tout leur salaire, alors qu'environ la moitié déclare n'avoir aucun contrôle sur son affectation.

Le chômage des 6-14 ans

Traditionnellement, les EE égyptiennes ne prennent en compte le chômage qu'à partir de 12 ans, si bien qu'on pourrait croire que c'est uniquement une affaire d'adultes ${ }^{21}$, mais cette idée est démentie par l'enquête, qui donne une image plus précise de ce phénomène, en particulier à 6-14 ans. Parmi les 1,387 million d'actifs de 6-14 ans, 78.000 ont déclaré être à la recherche d'un emploi, ce qui correspond à un taux de chômage de 5.62 \% chez les 6-14 ans, alors que le taux de chômage dans la population active âgée de 6 ans et plus était en Égypte de 7 \% pour la période de référence d'une 
semaine. Les taux de chômage respectifs des deux groupes d'âges distincts sont relativement proches - autour, de 5-6 \% (les 12-14 ans représentant les 3/5 des chômeurs de 6-14 ans). De plus, le chômage parmi les 6-14 ans touche presque de la même' manière les deux sexes (chômage masculin : 5,4 \% et féminin : $6 \%$ ).

Si, de 6 à 14 ans, le taux de chômage ne varie pratiquement pas selon le sexe ou le sousgroupe d'âge, les écarts selon le milieu sont importants. Pour ce qui est du taux de chômage global, il est en ville de 11,3 \% et de 4,2 \% à la campagne. Le taux de chômage augmente avec l'âge chez les urbains, alors qu'il reste stable et faible à la campagne.

Travail et scolarisation

Avant d'envisager les rapports entre travail et scolarisation, il faut tenter de faire le point sur l'importance de la catégorie (évoquée plus haut) des inactifs non scolarisés.

Les enfants de 6-14 ans ni actifs ni scolarisés

D'après les résultats inédits de cette enquête, les effectifs appartenant à la catégorie des inactifs non scolarisés sont loin d'être négligeables, et ce en dépit des efforts importants accomplis par l'Etat en matière de scolarisation. En effet, la proportion des enfants de 6-14 ans inactifs non scolarisés était estimée à $8 \%$ de la classe d'âge correspondante au moment de l'enquête (soit un effectif d'un peu moins d'un million), 7 \% des garçons de 6-14 ans répondant à ce cas de figure et $10 \%$ des dites. Si l'on considère les deux sousgroupes d'âge, les inactifs non scolarisés de 12-14 ans représentent $14 \%$ de leur classe d'âge contre $4 \%$ seulement chez les 6-11 ans. Ainsi, il y a tout lieu de croire que si la scolarisation des enfants en primaire remplit relativement bien son rôle, c'est à partir de l'entrée en préparatoire, soit à l'âge de 12 ans (et ce à la suite d'abandons pour diverses raisons, dont l'échec à l'examen final de cinquième année de primaire) que certains enfants quittent le système éducatif. S'agissant des différences entre les zones urbaines et les zones rurales (à l'intérieur desquelles l'enquête a distingué entre l'habitat villageois (HV) et l'habitat dispersé, (HD, hameaux), les différences dans la proportion des inactifs non scolarisés sont, comme on pouvait s'y attendre, significatives et en défaveur des jeunes filles. En effet, si en zones urbaines la proportion d'inactifs non scolarisés pour les deux sexes confondus n'est que de $3 \%$ ( $4 \%$ pour les garçons et $2 \%$ pour les filles) dans les zones rurales, plus défavorisées, elle passe à $11 \%$ dans l'HV et à $14 \%$ dans l'HD : dans les deux derniers cas, les filles inactives non scolarisées représentent respectivement $13 \%$ et $18 \%$, contre $8 \%$ et $10 \%$ des garçons. C'est sans conteste dans les HD que le pourcentage d'enfants de cette catégorie est le plus élevé et ce, particulièrement chez les filles de 12-14 ans où il atteint $28 \%$.

Il était tentant de rapprocher les effectifs des quatre catégories élémentaires entre lesquelles peuvent être répartis les enfants (ici de 6 à 11 ans) : scolarisés, actifs occupés, actifs à la recherche d'un emploi (" au chômage ») et inactifs non scolarisés.

Tableau 3 : Répartition de la population des 6-11 ans selon la scolarisation et le type d'activité (en milliers)

\begin{tabular}{|l|l|l|l|l|l|}
\hline & $\begin{array}{l}\text { Population } \\
\text { totale }\end{array}$ & $\begin{array}{l}\text { Population } \\
\text { scolarisée }\end{array}$ & $\begin{array}{l}\text { Population } \\
\text { active occupée }\end{array}$ & $\begin{array}{l}\text { Population au } \\
\text { chômage }\end{array}$ & $\begin{array}{l}\text { Population } \\
\text { inactive non } \\
\text { scolarisée }\end{array}$ \\
\hline \hline Garçons & 3.637 & 3.419 & 264 & 17 & 72 \\
\hline
\end{tabular}




\begin{tabular}{|l|l|l|l|l|l|}
\hline Filles & 3.364 & 2.691 & 268 & 12 & 202 \\
\hline
\end{tabular}

Source : ce tableau a été reconstitué en partie à partir des données de l'enquête emploi d'octobre 1988 et du recensement de population de 1986.

Les effectifs répondant à la première catégorie ont été calculés à l'aide des taux de scolarisation donnés par l'étude de N. Fergany (94\% pour les garçons, $80 \%$ pour les filles) appliqués aux effectifs de la classe d'âge des 6-11 ans estimés pour l'année 1988 (sur la base du RP 86); il en est de même pour les effectifs de la quatrième catégorie à l'aide des taux de garçons et filles inactifs non scolarisés donnés par l'EE 88. Les effectifs correspondant aux deuxième et troisième catégories sont ceux donnés directement dans les résultats de la même enquête. D'emblée, l'on peut constater que les effectifs cumulés des quatre catégories donnent, pour les garçons, un total supérieur de 135.000 à celui de la classe d'âge de 6-11 ans. Ces 135.000 garçons seraient donc à la fois des scolarisés et actifs, occupés pour leur très grande majorité et, sans doute, très marginalement " au chômage ». Ainsi, l'on peut avancer que presque $4 \%$ des garçons scolarisés entre 6 et 11 ans travaillaient, et que la moitié (51\%) des actifs occupés étaient scolarisés (en comptant pour négligeable le phénomène - possible - de la scolarisation des chômeurs).

S'agissant des jeunes filles, si le taux de scolarisation (80\%) est exact, ainsi que les effectifs d'activés occupées et de "chômeuses", on peut supposer que le taux de 6 \% d'inactives non scolarisées a très probablement été sous-estimé : les effectifs cumulés correspondant aux quatre catégories sont en effet inférieurs de 190.000 (très faible nombre) à ceux de la classe d'âge de 6-11 ans (féminine). En admettant qu'aucune fille de cet âge ne cumule scolarisation et travail, les inactives non scolarisées devraient être à peu près de 400.000 , ce qui correspondrait à un taux de près de $12 \%$ (et supérieur si les filles cumulent les deux activités). S'agissant des filles, on ne peut donc rien affirmer sur la proportion de celles qui travaillent et sont scolarisées à la fois.

Les enfants scolarisés : le cas des enfants travailleurs rémunérés

81 Grâce aux efforts déployés par l'Etat en matière de scolarisation, on a vu augmenter d'une année à l'autre les taux de scolarisation à 6-11 ans (tableau 4). Si cette augmentation quantitative de la scolarisation est importante, surtout chez les filles, elle ne reflète en rien la faible qualité des conditions de l'enseignement offert ${ }^{22}$.

Tableau 4 : Evolution du taux d'inscription dans l'ensemble du cycle primaire entre 198647 et 1992-93

\begin{tabular}{|l|l|l|l|l|l|l|l|}
\hline Année scolaire & $1986-87$ & $1987-88$ & $1988-89$ & $1989-90$ & $1990-91$ & $1991-92$ & $1992-93$ \\
\hline \hline Filles & 78,9 & 80,7 & 82,5 & 83,8 & 84,3 & 83,6 & 85,0 \\
\hline \hline Garçons & 95,1 & 96,0 & 97,3 & 98,0 & 98,3 & 97,0 & 97,9 \\
\hline \hline Total & 87,3 & 88,6 & 90,1 & 91,1 & 91,5 & 90,5 & 91,6 \\
\hline
\end{tabular}

Source : Ministère de l'Education nationale ; Education Planning and Information Division : Computing, Information and Statistics Directorale and the Education Planning Project, Research Triangle Institute, February 1994. 
82 enfants mais aussi, réciproquement, l'on attribue au travail des enfants la sousscolarisation, il semble intéressant de considérer pour elle-même, la relation entre travail et scolarisation. Les seules données disponibles (toujours dans l'enquête de 1988) permettant de faire la lumière sur cette relation de compatibilité/incompatibilité concernent la catégorie des enfants qui travaillent en dehors du cadre familial, catégorie qui, nous l'avons vu, représente $23 \%$ des enfants de 6-14 ans actifs occupés interrogés lors de l'enquête. Il ressort des résultats de l'enquête que plus de la moitié (soit $55 \%$ ) des enfants de 6-14 ans exerçant une activité rémunérée combinaient école et travail, ce qui va à l'inverse de l'idée communément admise selon laquelle le travail concurrence directement l'école. Les enfants travailleurs ayant prématurément quitté l'école représentaient $23 \%$ de cette catégorie, et ceux qui n'y avaient jamais eu accès 22 $\%$. Il faut noter d'importantes disparités selon la nature des emplois: en effet, sur l'ensemble des enfants de 6-14 ans occupés dans les emplois rémunérés agricoles, une proportion importante $(62 \%)$ travaillait et fréquentait l'école de façon concomitante. Ce pourcentage élevé peut s'expliquer par le fait que l'enfant en milieu rural peut être inscrit à l'école et ne pas la fréquenter de manière régulière, puisque l'obligation de présence n'est pas aussi rigoureuse que celle qui prévaut en milieu urbain. De plus, du fait du caractère saisonnier du travail agricole (entre juin et septembre, lors de la récolte du coton), il est plus facile aux enfants résidant en zone rurale d'avoir une activité scolaire tout en travaillant durant certains moments de l'année. A côté de ceux qui combinent les deux activités, les enfants travailleurs agricoles de 6-14 ans qui ont quitté l'école représentent $12 \%$, et ceux qui n'ont pas eu la chance d'y avoir accès $26 \%$.

83 A côté de ce premier type de profil, qui concerne les enfants occupés à des emplois agricoles, se dégage un deuxième profil correspondant davantage aux emplois non agricoles et prévalant en grande majorité en ville. En effet, parmi l'ensemble des enfants travailleurs occupés à ces emplois, quasiment la moitié (45\%) des enfants interrogés ont déjà quitté l'école ; il semblerait donc que la nature des emplois urbains offerts aux enfants ne soit en général que peu compatible avec l'activité scolaire ${ }^{23}$. Seulement $41 \%$ d'entre eux mènent de front leur activité productive et leur activité scolaire et $14 \%$ ne sont jamais allés à l'école (cela en proportion plus importante chez les garçons). On peut donc affirmer que le phénomène des abandons en cours de scolarité est plus important en milieu urbain ( $38 \%$ ) qu'en milieu rural (23\%). De plus, si en milieu urbain la catégorie des enfants qui n'ont jamais fréquenté l'école est de $10 \%$, elle est, en milieu rural, de $24 \%$.

列 on constate que mener de front les deux activités est plus fréquent chez les garçons (59 $\%)$ que chez les filles ( $42 \%$ ), que les abandons prématurés sont davantage le fait des garçons $(27 \%)$ que des filles (16\%) et que, sans grande surprise, le non-accès au système éducatif est plus important chez les filles (42\%) que chez les garçons (14\%).

Pour compléter ce tableau, il nous faut préciser, au terme de cette analyse, que dans la quasi-totalité des écoles, la journée scolaire est en réalité une demi-journée. Jusqu'ici en effet, nombre d'écoles fonctionnaient en deux périodes, voire trois dans la même journée, pratique qui permettait de doubler les effectifs scolarisés et qui, en outre, ménageait un temps pour le travail des enfants. Fait qui permettait également de rendre compatibles deux activités dont tout mène à croire qu'elles sont tout autant «formatrices». Or, les politiques affichées par le Ministère de l'Education, et qui se

Égypte/Monde arabe, 18-19 | 1994 
situent dans une grande entreprise de réhabilitation de l'enseignement public, visent désormais à instaurer progressivement la journée scolaire pleine dans tous les établissements d'ici 1997. Reste à savoir quels seront les effets de cette politique sur le travail des enfants. Si le choix entre scolarisation et travail s'impose, sera-t-il favorable à l'école? Reste également à poser la question des représentations sous-jacentes aux politiques d'enseignement. Le volontarisme d'Etat, dans ce domaine, peut-il imprimer sa marque sur la recomposition des pratiques sociales? En définitive, au travers de la relation travail/école, la question posée est, certes, celle de la formation, mais plus fondamentalement celle des modes de socialisation.

\section{NOTES}

1. Je remercie le docteur Nader Fergany et Mohammed Hadari pour l'aide précieuse qu'ils ont bien voulu m'apporter. N. Z.

2. Aries Ph., L'enfant et la vie de famille sous l'Ancien Régime, Editions du Seuil, 1973.

3. Bangnee A. L., Manuel de méthodologie, UNESCO, Nations Unies.

4. CAPMAS, Central Agency for Public Mobilization and Statistics.

5. Fergany N., « A Characterization of the EmploymenI Problem in Egypt », in Employment and Structural Adjustment : Egypt in the 1990s, H. Handoussa and G.Potier, The American University in Cairo Press, 1991. p. 25 sq.

6. Selon M. Nagt, « This is an entirely normal situation in a country where a relatively unmechanized economy exists, especially in agriculture and the services, compulsory school atlendance is not achieved, and population pressure accompagnied by an age structure skewed heavily toward the younger ages ", In « Child Labor in Rural Egypt », Rural Sociology, vol. 37, n 4, December 1972.

7. Au RP 17, dont la notice explicative est très complète, sont par exemple regroupés sous celle rubrique des marchands, des négociants et des commerçants sans autre spécification.

8. Nassef A. F., The Egyptian Labor Force: Its Dimensions and Changing Structure, 1907-1960, University of Pennsylvania. Population Studios Center, 1970.

9. Nassef A. F., op. cit., p. 41 : « The fluctuations in the rate of total labor force growth may be accounted for mainly by inconsistences in reporting of economically actives young persons and the ill-defined group. Exclusion of these groups does not mean that all their members were not in the labour force, but that the degrees to which they were covered in the enumeration of economically active persons varied in the different censuses. It is preferable to omit them or deal with them separately rather than to make any gross adjustments of the data based on arbitrary assumptions about the extent of coverage. "

Par ailleurs et à propos du même problème, D. Mead déclare : «We have found it best to exclude these people from our employment statistics, for a number of reasons. Seventy-five percent of this group in « ill-defined industries » were in the provinces (rural), and the largest number were in the age group 10-29. If this group is included as employed, the age-specific participation rates looks unreasonably high, compared to earlier and later years. It seems plausible to assume that this group is made up primarily of men who have no regular employment, but who are happy to 
take any temporary job they can find. ", Growth and Structural Change in the Egyptian Economy, Homewood, Illinois, USA publication of the Economic Growth Center, Yale University, 1967, p. 38. 10. II est intéressant de noter que la circulaire $n^{\circ} 13$ intitulée « Instructions aux agents recenseurs » du RP 17 stipule, à propos de la question sur la profession: « II ne faut pas négliger d'enregistrer la profession des femmes et des enfants, surtout lorsqu'il s'agit de familles de paysans dans lesquelles femmes et enfants prennent part aux travaux agricoles. "

11. Nasser: « The census data are not completely comparable over time. Changes in concepts and Systems of classification have been taken place. Although no information about the changes in concepts and classifications is published in the census reports », 1970, p. 8.

12. L'on trouverait probablement autant d'affirmations d'une diminution du phénomène (basée sur l'argument de la scolarisation et du déclin du travail des enfants dans l'agriculture) que de sa croissance (basée sur l'augmentation du coût de la vie, de la crise...).

13. Cleland W. affirmait : "Egyptian have been counted more times than any other people ", The Population Problem in Egypt. Lancaster Science Press Printing Co., 1936.

14. L'on peut observer que même en prenant l'année 1977 comme point d'inflexion, on constate malgré tout une multiplication par 2,6 du TA et par 3,5 des effectifs. La remontée semble donc réelle et importante.

15. CAPMAS, Results of the Labour Force Sample Survey, Fourth Quarter 1988, detailed employment characteristics, December 1990.

16. Fergany N., Child Labour in the Arab Countries, prepared for the Arab Council for Childhood and Development, September 1993 : «The estimate of the size of child labour varies between population censuses, the regular series of the LFSS and the exceptional October 1988 round of the LFSS, as a result of differences in definitions, field procedures and the quality control measures. Definitions could differ, even within the same country, in the extent of economic activity, the lower age limit, the length of the reference period and the minimum duration of work required to count a person as economically active. Differences in the timing of statistical operations impede comparability among different sources as a result of the seasonality of the economic activity, particularly in the case of children ».

17. "A multi-stage stratified random sample of 10.000 housing units. "

18. Outre le traditionnel questionnaire portant sur les ménages, l'enquête a donné lieu à la conception et à la mise en place d'une batterie de modules individuels concernant des questions d'ordre général telles que le chômage, le sous-emploi, la mobilité, la migration de travail ainsi que les revenus, qui avaient pour objectif d'intégrer un projet intitulé « Labour Information System ». C'est également dans le cadre de ce projet que des éludes spécialisées concernant les récentes évolutions du marché de l'emploi dans les secteurs de l'agriculture, de la construction, de l'informel ainsi que du secteur industriel ont été réalisées. Cf. Fergany N., Final; Report, Overview and General Features of Employment in the Domestic Economy, April 1991.

19. Parmi les trois périodes de référence, la semaine a été retenue ici pour assurer la comparabilité avec les résultats des enquêtes antérieures. Il suffit que la personne ait travaillé une heure durant la période précédant l'enquête pour être considérée comme active.

20. Fergany N., Child Labour in the Arab Countries, 1993, p.23.

21. Le taux de chômage des 15-64 ans était, d'après l'enquête d'octobre 1988 , de 7,18 \%, ce qui correspond à 1,239 million.

22. Une étude portant sur les écoles primaires gouvernementales mentionne que $7 \%$ des écoles primaires publiques ne disposent d'aucune source d'eau potable, $48 \%$ n'ont pas de toilettes en bon état et 3,5\% n'en ont pas du tout ; enfin $60 \%$ n'ont pas d'électricité, National Specialized Council, 1979, The Amendment of Primary Education. 
23. Measures of the Efficiency of Primary Education in Egypt, 1985/1986 to 1991/1992, prepared by Ministry of Education and the Education Planning Project, Research Triangle Institute, December 1993. Selon cette étude portant sur l'enseignement primaire en Égypte, le taux d'abandon des 2 sexes était, en cours d'année de la cinquième année du primaire, de 6,6\%, avec un taux plus élevé chez les garçons que chez les filles : soit respectivement 7,2 \% et 5,9 \% pour l'année scolaire 1991-92.

INDEX

Mots-clés : éducation, enseignement, scolarisation, statistiques, travail, travail des enfants, enfants

\section{AUTEUR}

NADIA ZIBANI

CEDEJ 